\title{
NOTAS
}

\section{Notas Sobre El "Tablero de Dirección" en Rayuela de Julio Cortázar}

La mayoría de los críticos de Julio Cortázar parecen haber entendido mal lo del "Tableto de dirección" que se encuentra en la primera página de Rayuela. Antes de tratar de aclarar el propósito de dicho tablero, creo que conviene reproducirlo, tal como aparece en la novela, para los que no lo tengan a mano:

\section{Tablero de Dirección}

A su manera este libro es muchos libros, pero sobre todo es dos libros. El lector queda invitado a elegir una de las dos posibilidades siguientes:

El primer libro se deja leer en la forma corriente, y termina en el capítulo 56 , al pie del cual hay tres vistosas estrellitas que equivalen a la palabra Fin. Por consiguiente, el lector prescindirá sin remordimientos de lo que sigue.

El segundo libro se deja leer empezando por el capítulo $73 \mathrm{y}$ siguiendo luego en el orden que se indica al pie de cada capitulo. En caso de confusión u olvido, bastará consultar la lista siguiente: $73-1-2-116-3-84-4-71-5-81-74-6-7-8-$ $93-68-9-104-10-65-11-136-12-106-13-115$ $14-114-117-15-120-16-137-17-97-18-153$ $19-90-20-126-21-79-22-62-23-124-128-24$ $134-25-141-60-26-109-27-28-130-151-152-143$ $100 \cdot 76-101-144-92-103 \cdot 108-64-155-123-145$ 


$$
\begin{aligned}
& 122-112-154-85-150-95-146-29-107-113 \\
& 30-57-70-147-31-32-132-61-33-67-83-142 \\
& 34-87-105-96-94-91-82-99-35-121-36-37-98 \\
& 38-39-86-78-40-59-41-148-42-75-43-125-44 \\
& 102-45-80-46-47-110-48-111-49-118-50-119 \\
& 51-69-52-89-53-66-149-54-129-139-133-140 \\
& 138-127-56-135-63-88-72-77-131-58-131 .
\end{aligned}
$$

Con objeto de facilitar la rápida ubicación de los capítulos, la numeración se va repitiendo en lo alto de las páginas correspondientes. a cada uno de ellos. ${ }^{1}$

Muchos críticos ( $y$ sin duda muchos lectores) han tomado a broma las dos maneras de leer sugeridas por Cortázar y han leído la novela entera en la forma tradicional (i.e., desde el capitulo 1 hasta el capítulo 155, desatendiendo por completo el tablero). Frecuentemente se han sentido obligados a leer la novela de las dos maneras señaladas por el autor. H. A. Murena escribe, por ejemplo: "Quien escribe estas líneas se sintió en el deber... de practicar la lectura del libro en los dos sentidos que el autor indica... y tras de apelar a toda su paciencia..."2 Juan Carlos Ghiano escribe de igual manera: "He leído Rayuela con los dos métodos indicados; me he impuesto la segunda lectura..."3 E. D. Carter ha afirmado asimismo que por motivo del tablero, Rayuela "must be read at least twice."4

Cortázar mismo ha escrito con respectó a este error:

En Rayuela definí y ataqué al lector-hembra, al incapaz de la verdadera batalla amorosa con una obra que sea como el ángel para Jacob. Si se dudara de la legitimidad de mi ofensiva, baste este ejemplo: críticos reputados con sede en Buenos Aires emṕezaron por no entender el doble sistema posible de lectura de la novela, y de ahí pasaron al pollice verso después de asegurar patéticamente que la habían leído de las dos maneras que indica el autor, cuando

1 Julio Cortázar, Rayuela (Buenos Aires: Editorial Sudamericana, 1967), 64 edición).

2 H. A. Murena, "Julio Cortázar: Rayuela", Czadernos, No. 79 (diciembre, 1963), p. 86.

Juan Carlos Ghiano, La Nación (Buenos Aires), Suplemento Literario, 20 de octubre, 1963 , p. 5.

4 E. D. Carter, "Magical Realism in Contemporary Argentine Fiction", Doftoral Dissertation, University of Southern California, 1966, p. 353. 
lo que proponia el pobre autor era una opción y jamás hubiera tenido la vanidad de pretender que en nuestros tiempos se leyera dos veces un mismo libro. ${ }^{5}$

El verdadero propósito del "Tablero de dirección" es distinguir al lector activo, creativo (el "lector cómplice") del lector pasivo, tradicional (el "lector-hembra"). Cortázar busca un lector que esté dispuesto a participar activamente en la creación (o "re-creación") de la novela $y$ en el destino de los personajes:

Posibilidad tercera: la de hacer del lector un cómplice, un camarada de camino. Simultaneizarlo puesto que la lectura abolirá el tiempo del lector y lo trasladará al del autor. Así el lector podría llegar a ser copartícipe y copadeciente de la experiencia por la que pasa el novelista... está operando un misterio que el lector cómplice deberá buscar... Lo que el autor de esa novela haya ligrado para sí mismo, se repetirá (agigantándose, quizá, y eso sería maravilloso) en el lector cómplice...

Aplicando esta distinción entre "lector-hembra" y "lector cómplice" al problema del tablero, es claro que el primer requisito para incluirse en esta última categoría es el de tomarse la molestia de leer la novela de la segunda manera indicada por el autor (i.c., según la lista incluida en el tablero). Leyendo Rayuela de esta manera, el lector alterna capitulos de las dos primeras partes del libro con los de la tercera parte ("Capítulos prescindibles"). De este modo el lector experimenta una continua serie de saltos entre lo vital, representado por las aventuras y desventuras del protagonista, Horacio Oliveira, en París (primera parte) y luego en Buenos Aires (segunda parte), y lo abstracto de la tercera parte consiste principalmente en las tecrías metafísicas y literarias del autor, disfrazado aquí en la persona del ficticio teórico literario, Morelli.

El lector que elige la primera manera de aproximarse al texto sugerida por Cortázar sacrifica por completo la lectura de los capítulos de la tercera parte, la cual, además de ser una significativa adición a las dos primeras, es muchas veces la clave para su interpretación. También se engaña el lector que cree poder enterarse del contenido de la novela leyéndola a la manera tradicional. Esta última manera de leer Rayuela (que es sin duda la más frecuente entre los que solamente leen la novela

- Julio Cortázar, La vuelta al dia en ochenta mundos (México: Siglo Veintiuno Editores, 1967), P. 142.

- Cortázar, Rayuela, pp. 453-454, 
una vez) presupone que el orden de los "Capítulos prescindibles" sea arbitrario. Pero éste no es el caso.

Para substanciar que el orden de los "Capítulos prescindibles" no sea arbitrario (tal como alternan con los primeros capítulos en la lista indicada en el tablero), bastará demostrar una relación lógica entre estos capítulos y los de las dos primeras partes. Aunque tal relación no sea siempre muy obvia, hay varios casos en que la conexión entre los capitulos es bastante clara. Mencionaremos solamente tres:

1) Hay una relación explícita entre el capitulo 14 en que Wong muestra a los amigos del "Club de las serpientes" las fotografías de una ejecución a cuchillo de un hombre en Pekín y el capítulo 114 que consiste en un recorte de periódico norteamericano que describe la muerte de un asesino ejecutado en la cámara de gas de la prisión de San Quintín.

2) La relación entre el capítulo 15 y el capitulo 120 es un poco menos clara. En el capítulo 15 la amante de Horacio Oliveira, la Maga, cuenta a sus amigos la historia de su violación por un negro cuando tenía trece años. El capítulo 120 trata de un recuerdo infantil de una persona llamada Ireneo. El recuerdo consiste en la imagen de unas hormigas que tratan de meter un gusano en su hormiguero. La relación simbólica entre los dos capitulos se hace más explícita en el capítulo 16 cuando resulta que el nombre del violador de la Maga también fue Ireneo.

3) A veces alterna un capítulo de tono patético, o aún trágico, con otro de tono más bien burlesco, como en los capítulos 28 y 137. Después del largo capítulo 28 en que Horacio y sus amigos pasan la noche hablando, bebiendo y escuchando viejos discos de jazz, mientras el hijo de la Maga, Rocamadour, se está muriendo en el mismo cuarto, pasamos al capitulo 137, que consiste en un recorte de The Observer de Londres titulado "Riesgos del cierre relámpago." Este recorte es una advertencia contra una "nueva clase de accidente que pueden sufrir los niños" que consiste "en que el prepucio se quede atrapado por el cierre".

Los otros ejemplos de una relación concreta entre los capítulos de la tercera parte y los de las dos primeras - siguiendo siempre el orden que sugiere el autor- son bastante numerosos para eliminar toda posi- 
bilidad de coincidencia casual. También es claro que los capitulos que Cortázar llama "prescindibles" no lo son de ninguna manera. Además de ser muchas veces la interpretación abstracta de la acción de las dos primeras partes, estos capítulos completan algunas veces esta misma acción. Aunque la mayoría de los capítulos de la tercera parte consisten en recortes de periódicos y citas de varios escritores (tal como José Lezama Lima, Meister Eckhart, Claude Lévi-Strauss, Octavio Paz, y sobre todo el teórico literario, Morelli), algunos son como huétfanos aislados y extraviados de las dos primeras partes. Estos últimos capítulos contienen las divagaciones de Horacio Oliveira $\mathrm{y}$ sus amigos, y continúan e incluso adelantan $\mathrm{l}_{a}$ acción de las primeras partes. Un excelente ejemplo de este proceso son los capítulos 135 - 63 - 88 - 72 - 77 - 131 - 58, es decir, los que prosiguen con la acción de la novela después del último capítulo de la segunda parte. Solamente en estos capítulos nos enteramos de que Oliveira todavía está vivo después de su caída por la ventana del manicomio en el captíulo 56. Por lo tanto, el que no lee estos capítulos de la tercera parte se queda con la falsa impresión de que Oliveira se haya suicidado. Otros capítulos de la tercera parte, como el 57 y el 76 , suplementan de una manera semejante la acción de la primera parte del libro.

Ahora bien, si el orden de los capítulos sugerido por el autor es deliberado, y si los capítulos de la tercera parte completan los de las dos primeras, tenemos que concluir que el "Tablero de dirección" que nos of rece Cortázar no es algo que debamos tomar a la ligeta. Conocida es la pasión que tiene Cortázar por los juegos. Tanto la rayuela misma (como se juega en la Argentina y en Europa) como el mandala (el símbolo budista que Cortázar pensaba en un principio utilizar como título de la novela) son concebidos por Cortázar como juegos metafísicos, iniciativos, de aplicación existencial. Los que conocen la natrativa de Cortázar saben que su autor concibe la vida humana en términos de una búsqueda de un orden superior. Tanto Horacio Oliveira en Rayuela como Johny Carter en "El perseguidor", Medrano en Los premios y Juan y los otros en 62. Modelo para armar, son buscadores de la verdad, perseguidores de una realidad más profunda y auténtica. En Rayuela esta búsqueda, concebida en términos metafísicos, literatios y existenciales, se puede ver en los más diversos niveles. Los personajes, y especialmente Horacio Oliveira, buscan nuevas dimensiones de conciencia a través del amor, de la música, del intelecto, de la literatura, de lo absurdo. El propio Cortázar busca por medio de sus personajes, del acto creativo de escribir y del lenguaje. Pero Cortázar no se satisface con estas limi- 
taciones sino que insiste en que el lector participe asimismo en la búsqueda del centro, de la última casilla de la rayuela, del cielo, avanzando en su propio mandala a medida que toma parte en este mundo de la imaginación, del juego. El significado del "Tablero de dirección" en Rayzela tiene que ser definido teniendo en cuenta esta preocupación de Cortázar por el lector. Si el tablero es en cierto sentido un juego, la intención del autor es que se tome este juego muy en serio. El que lo haga, el que decida ser "lector cómplice", entrando en el laberinto cortazariano, puede ser que llegue a una perspectiva más amplia de la literatura y de la realidad, que es precisamente lo que quisiera el autor.

University of Wisconsin-Parkside. 